\title{
Transmission Capacity of Decode-and-Forward Cooperation in Overlaid Wireless Networks
}

\author{
Zhengguo Sheng*, Zhiguo Ding†, Kin K Leung* \\ *Department of Electrical and Electronic Engineering, Imperial College, UK \\ $\dagger$ Department of Communication Systems, Lancaster University, UK \\ Email: zhengguo.sheng06@imperial.ac.uk
}

\begin{abstract}
In this paper, we employ a stochastic geometry model to analyze the transmission capacity of the Decode-andForward (DAF) cooperation scheme in an overlaid wireless network where a primary (PR) network and a secondary (SR) network coexist together. The PR users employ DAF scheme and have a higher priority to access the channel, whereas the SR users use only direct transmission. Because of the fact of coexistence, the interference from SR network seriously affects the performance of PR network. Assuming that simultaneous transmitters in both networks are randomly located in space according to Poisson point processes, we develop outage probabilities for both DAF and direct transmission schemes in both deterministic and Rayleigh fading channels. By defining transmission capacity in terms of the outage probability, a desired data rate and the density of transmissions, we further quantify transmission capacities for both schemes. It shows that the use of cooperative transmission achieves much better reliability and a larger transmission capacity than the use of direct transmission in the PR network. Furthermore, such performance gain can be manipulated to increase the transmission capacity of the SR network without deteriorating the performance of the PR network. Numerical results also demonstrate the significant improvement on the transmission capacity by using cooperative transmission.
\end{abstract}

\section{INTRODUCTION}

Cooperative transmission (CT) has recently attracted much attention as an effective technique to combat multi-path fading and enhance receiver reliability in wireless communication systems. The key feature of cooperative transmission is to encourage single-antenna devices to cooperatively share their antennas such that a virtual antenna array can be constructed, thereby, enabling performance to be significantly boosted. However, existing work more focuses on reception reliability (i.e., BER) and energy issues; the potential of the transmission capacity of cooperative networks has not been fully explored.

There have been some state-of-art works on ad hoc network capacity [1]-[4]. Gupta and Kumar establish the transport capacity in their pioneer work [1] and show that it can be best achieved as $\Theta(\sqrt{\lambda})$, where $\lambda$ is the density of transmissions. In order to further explore the relation between the transmission capacity and other system parameters, e.g., channel model,

This research was sponsored by US Army Research laboratory and the UK Ministry of Defence and was accomplished under Agreement Number W911NF-06-3-0001. The views and conclusions contained in this document are those of the authors and should not be interpreted as representing the official policies, either expressed or implied, of the US Army Research Laboratory, the U.S. Government, the UK Ministry of Defense, or the UK Government. The US and UK Governments are authorized to reproduce and distribute reprints for Government purposes notwithstanding any copyright notation hereon.
MAC scheduling and power consumption, Weber et al. in [2] derive simple expressions for both upper and lower bounds on the transmission capacity using a stochastic geometry approach. Taking one step further, Weber et al. [3] and Toumpis et al. [4] address the impact of channel fading on the network capacity and argue that fading can actually reduce the transmission capacity. All the above results only focus on direct transmission. However, this fundamental problem for a general cooperative network is still open.

Meanwhile, due to the fact that most of the available spectrum is poorly utilized which causes a shortage of spectrum for new wireless services, cognitive radio has emerged as a promising technique to enable secondary (SR) networks to coexist with licensed primary (PR) networks. In such an overlaid wireless network, PR users have a higher priority to access the spectrum and SR users need to operate conservatively such that the interference does not negatively affect the service quality of PR network. Motivated by [5] that the total transmission capacity of the two networks (PR and SR networks) can be significantly boosted over that of a single network, we further introduce a cooperative transmission scheme into overlaid wireless networks and investigate its performance gain on transmission capacities in both PR and SR networks.

The contribution of this paper is two-fold: first, we investigate the average interference performance through the stochastic geometry model and then use it to obtain outage probabilities and transmission capacities for both direct transmission and the Decode-and Forward (DAF) cooperation scheme. Second, we show that such performance gain can be manipulated to increase the transmission capacity of the SR network without deteriorating the performance of PR network.

\section{SySTEM MODEL}

We consider an overlaid wireless network in Fig. 1 where a network of PR users and a network of SR users coexist in the same physical region. The primary network employs cooperative transmission and since it is a licensed network, PR users have a higher priority to access the channel. Whereas in the secondary network, unlicensed SR users use only direct transmission to communicate. Due to the fact of coexistence, interference from one network will affect another. Especially, we are interested in the interference brought from SR users to PR users. The motivation for introducing cooperative transmission into the primary network is to further achieve performance gain that cooperative transmission can bring to PR users as well as the benefits brought to SR users. 


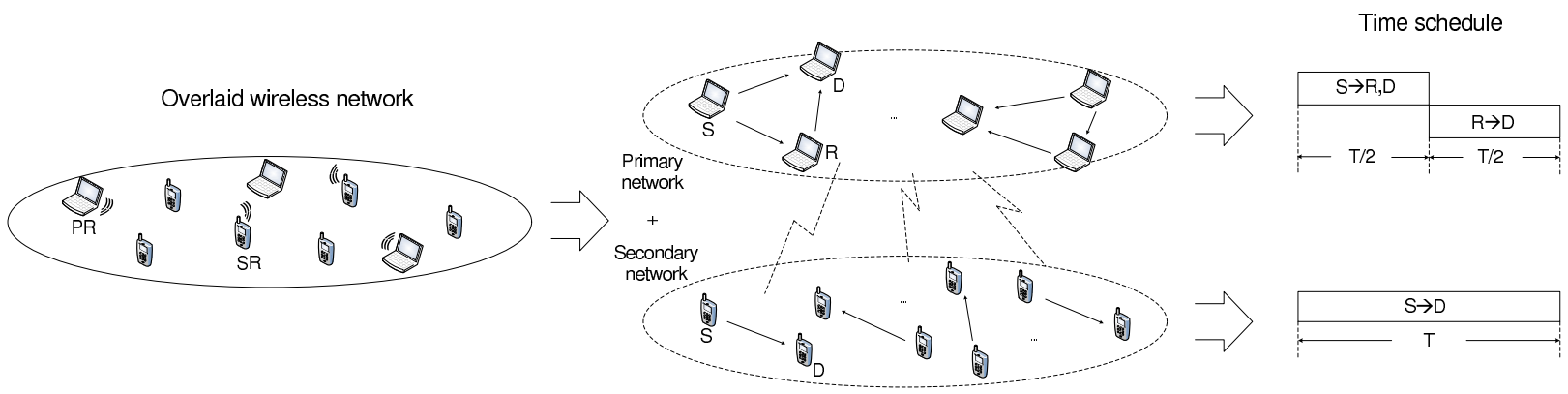

Fig. 1. One example of overlaid wireless network

Both deterministic and Rayleigh fading channels are considered. The co-channel interference behavior is clearly presented by the stochastic geometry model. Simultaneous transmitters in both PR and SR networks are modeled by two independent homogeneous Poisson point process with density $\lambda_{\mathrm{PR}}$ and $\lambda_{\mathrm{SR}}$, respectively. In order to simplify the interference behavior, we assume $\lambda_{\mathrm{PR}} \ll \lambda_{\mathrm{SR}}$, where the interference is dominated by simultaneous SR transmitters in the network. This is a sensible assumption, because the number of PR transmitters is expected to be much less than that of SR transmitters due to high admission requirements to limit the number of PR users to ensure their reception quality. In the rest of paper, we simply use $\lambda_{\mathrm{I}}$ to denote the density of simultaneous interferers.

In order to evaluate the performance of a more practical distributed network, we assume that all the transmitters are using ALOHA-type [6] transmission without centralized scheduling. However, time schedules between two networks are fully synchronized. Compared with the time schedule of direct transmission in Fig. 1, cooperative transmission shares one time slot (T) with two orthogonal channels and has a source transmitting its signals to both relay and destination in one phase (T/2), and then the relay forwarding the signals to the destination in a second phase. The destination can employ a variety of techniques to combine and decode the received signals from both the source and relay.

\section{TRAnSmission CAPACITY FOR Deterministic CHANNEL}

In this section, we first derive the capacity of cooperative transmission and then compare its performance with direct transmission. We assume that all PR and SR transmitters within the network use the same transmission power $P_{t}$ to transmit over the same distance $d$ between a source and a destination and $P_{n}$ is the noise power. The wireless link between the nodes $i$ and $j$ is modeled as $\mathrm{a}_{i j}=d_{i j}^{-\alpha / 2}$, where $d_{i j}$ is the distance between the nodes $i$ and $j$, represents the large-scale behavior of the channel gain and $\alpha$ is the path-loss exponent.

\section{A. Direct Transmission}

As compared with cooperative transmission, we start our analysis with a simple transmission strategy where direct transmission is adopted by PR users. To establish a baseline performance for direct transmission, the maximum average mutual information between the source and the destination in this network scenario is given by

$$
\mathrm{I}_{D}=\log \left(1+\frac{P_{t} d_{s, d}^{-\alpha}}{P_{n}+\sum_{i \in \Phi} P_{t}\left|X_{i}\right|^{-\alpha}}\right)
$$

where the interference is dominated by SR transmitters. In order to simplify the notation, we directly use $\Phi$ to represent the set of simultaneous interferers from SR users. The outage event for a desired transmission data rate $R$ in bit/s/Hz is given by $\mathrm{I}_{D}<R$ and the outage probability satisfies

$$
\begin{aligned}
& \operatorname{Pr}\left[\mathrm{I}_{D}<R\right]=\operatorname{Pr}\left[\frac{P_{t} d_{s, d}^{-\alpha}}{P_{n}+\sum_{i \in \Phi} P_{t}\left|X_{i}\right|^{-\alpha}} \leq 2^{R}-1\right] \\
& =\operatorname{Pr}\left[\frac{P_{n}}{P_{t}}+\sum_{i \in \Phi}\left|X_{i}\right|^{-\alpha} \geq \frac{d_{s, d}^{-\alpha}}{2^{R}-1}\right]=\operatorname{Pr}[X \geq \varepsilon],
\end{aligned}
$$

where $X=\sum_{i \in \Phi}\left|X_{i}\right|^{-\alpha}$ and $\varepsilon=\frac{d_{s, d}^{-\alpha}}{2^{R}-1}-\frac{P_{n}}{P_{t}}$. According to [7], the moment generating function (MGF) of $X$ is derived as

$$
\Phi_{X}(s)=\exp \left(-\pi \lambda_{\mathrm{I}} s^{\frac{2}{\alpha}} \Gamma\left(1-\frac{2}{\alpha}\right)\right),
$$

where $\Gamma(z)=\int_{0}^{\infty} t^{z-1} e^{-t} d t$ is incomplete gamma function and $\lambda_{\mathrm{I}}$ is the density of interferers. When $\alpha=4$, we can get a closed form expression for probability density function (PDF) of $X$ via inverse Laplace transform

$$
f_{X}(x)=\frac{\pi}{2} \lambda_{\mathrm{I}} x^{-\frac{3}{2}} \exp \left(-\pi^{3} \frac{\lambda_{\mathrm{I}}^{2}}{4 x}\right), \quad x \geq 0
$$

then the corresponding cumulative density function (CDF) of $X$ is

$$
F_{X}(x)=\operatorname{erfc}\left(\frac{\pi^{\frac{3}{2}} \lambda_{\mathrm{I}}}{2 \sqrt{x}}\right)=2 Q\left(\frac{\pi^{\frac{3}{2}} \lambda_{\mathrm{I}}}{\sqrt{2 x}}\right),
$$

where $\operatorname{erfc}$ is the complementary error function. So the outage probability for direct transmission (DT) is

$$
p_{D T}^{\text {out }}=\operatorname{Pr}[X \geq \varepsilon]=1-2 Q\left(\frac{\pi^{\frac{3}{2}} \lambda_{\mathrm{I}}}{\sqrt{2 \varepsilon}}\right)
$$

and the transmission capacity of PR users using direct transmission is given by

$$
C_{D T}=R \lambda\left(1-p_{D T}^{\text {out }}\right) .
$$

where $R$ is a desired transmission data rate, $\lambda$ is average density of successful simultaneous transmissions and $P^{\text {out }}$ is outage probability. 


\section{B. Cooperative Transmission}

The PR users actually employ the Decode-and-Forward (DAF) transmission scheme which allows the relay to decode the signals from the source, re-encode and retransmit the signals to the destination. Specifically, we consider here the Selection Decode-Forward scheme [8]. If the relay node cannot successfully decode the signals from the source, the source simply repeats its transmission directly to the destination (same as direct transmission); otherwise, the relay forwards what it received from the source using decode-forward.

Let $d_{s, d}, d_{s, r}$ and $d_{r, d}$ be the respective distances among the source, relay and destination. During the first phase (T/2), the destination receives $y_{d}=\frac{x_{s}}{d_{s, d}^{\alpha / 2}}+n_{d}$ from the source node, where $x_{s}$ is the information transmitted by the source and $n_{d}$ is white noise. During the second phase, the destination node receives

$$
y_{d}= \begin{cases}\frac{x_{s}}{d_{s, d}^{\alpha / 2}}+n_{d}, & \text { if } \operatorname{SINR}_{s, r}<q(R) \\ \frac{x_{r}}{d_{r, d}^{\alpha / 2}}+n_{d}, & \text { if } \operatorname{SINR}_{s, r} \geq q(R)\end{cases}
$$

where $q(R)=\left(2^{2 R}-1\right)$ can be derived from direct transmission and is analogous to (1). As can be seen from the first condition of (8), when the link between the source and the relay is so poor that the relay is not able to decode, there is no diversity gain can be achieved and the source is repeating its transmission during this phase. The second condition corresponds to the case when the relay can decode and repeat the source transmission, thus obtaining the secondorder diversity gain through CT. Therefore, choosing a proper relay to guarantee the link quality is critical in achieving good system performance.

A closed form expression of outage probability for cooperative transmission can be obtained as

$$
\begin{aligned}
p_{C T}^{\text {out }}=\quad & \left(1-2 Q\left(\frac{\pi^{\frac{3}{2}} \lambda_{\mathrm{I}}}{\sqrt{2 \varepsilon_{1}}}\right)\right)\left(1-2 Q\left(\frac{\pi^{\frac{3}{2}} \lambda_{\mathrm{I}}}{\sqrt{2 \varepsilon_{2}}}\right)\right) \\
& +2 Q\left(\frac{\pi^{\frac{3}{2}} \lambda_{\mathrm{I}}}{\sqrt{2 \varepsilon_{1}}}\right)\left(1-2 Q\left(\frac{\pi^{\frac{3}{2}} \lambda_{\mathrm{I}}}{\sqrt{2 \varepsilon_{3}}}\right)\right) .
\end{aligned}
$$

where $\varepsilon_{1}=\frac{d_{s, r}^{-\alpha}}{2^{2 R}-1}-\frac{P_{n}}{P_{t}}, \varepsilon_{2}=\frac{2 d_{s, d}^{-\alpha}}{2^{2 R}-1}-\frac{P_{n}}{P_{t}}$ and $\varepsilon_{3}=$ $\frac{d_{s, d}^{-\alpha}+d_{r, d}^{-\alpha}}{2^{2 R}-1}-\frac{P_{n}}{P_{t}}$. The mathematical detail of (9) is provided in Appendix-A and the transmission capacity of PR users using cooperative transmission is given by

$$
C_{C T}=R \lambda\left(1-p_{C T}^{o u t}\right) .
$$

Theorem 1: For a receiver in the PR network, the reliability gain $g$, which is defined as the ratio of outage probability achieved by using cooperative transmission to that achieved by using direct transmission, is shown as

$$
g=\sqrt{\frac{d_{s, d}^{-\alpha}\left(2^{R}+1\right)}{d_{s, d}^{-\alpha}+d_{r, d}^{-\alpha}}} .
$$

Proof: See Appendix-B.

It is worth noting that (11) can be achieved only when a small data rate $R$ is applied to achieve a low error rate. In general, Theorem 1 tells us the use of cooperative transmission achieves much better reliability (outage performance) than use of direct transmission. For example, for the case where the distance between a source and a destination $d_{s, d}=1$, the relay is in the middle between the source and destination $d_{r, d}=$ 0.5 , the data rate $R=1 \mathrm{bit} / \mathrm{s} / \mathrm{Hz}$ and the path loss exponent $\alpha=4$, the reliability gain $g$ is 0.42 , which means the outage probability can be reduced up to $58 \%$ for PR users when using cooperative transmission. Such reliability gain can be further improved when the relay closes to the destination and will be verified by numerical results in Section $\mathrm{V}$.

Theorem 2: In achieving the same QoS requirement, using cooperative transmission in PR network can significantly increase the number of simultaneous SR transmitters by $1-\sqrt{\frac{d_{s, d}^{-\alpha}\left(2^{R}+1\right)}{d_{s, d}^{-\alpha}+d_{r, d}^{-\alpha}}}$, as compared with the primary network using direct transmission.

Proof: Due to the space limitation, the proof is omitted here and can be referred from the similar method in Appendix-B.

\section{TRANSMISSION CAPACITY FOR RAYLEIGH FADING CHANNEL}

In order to find the channel fading impact on network capacity, we further derive transmission capacities for two schemes under a fading channel scenario. The channel model is assumed as $\mathrm{a}_{i j}=\frac{h_{i j}}{d_{i j}^{\alpha / 2}}$, where $h_{i j}$ captures the channel fading characteristics due to the rich scattering environment and is assumed as independent and identically distributed (i.i.d), complex Gaussian variable with zero mean and unit variance.

\section{A. Direct Transmission}

The outage probability for direct transmission can be derived as

$$
\begin{aligned}
\operatorname{Pr}\left[\mathrm{I}_{D}<R\right] & =\operatorname{Pr}\left[\frac{P_{t} d_{s, d}^{-\alpha}\left|h_{s, d}\right|^{2}}{P_{n}+\sum_{i \in \Phi} P_{t}\left|X_{i}\right|^{-\alpha}\left|h_{i, d}\right|^{2}} \leq 2^{R}-1\right] \\
& =\operatorname{Pr}\left[\left|h_{s, d}\right|^{2} d_{s, d}^{-\alpha} \leq\left(2^{R}-1\right)\left(\frac{P_{n}}{P_{t}}+X\right)\right],(12)
\end{aligned}
$$

where $X=\sum_{i \in \Phi}\left|X_{i}\right|^{-\alpha}\left|h_{i, d}\right|^{2}$. Since $\left|h_{i, j}\right|^{2}$ is an exponential random variable with unit mean, the moment generating function (MGF) of $X$ is derived as

$$
\begin{aligned}
\Phi_{X}(s) & =\exp \left(-\pi \lambda \mathbb{E}\left[h^{\frac{2}{\alpha}}\right] s^{\frac{2}{\alpha}} \Gamma\left(1-\frac{2}{\alpha}\right)\right) \\
& =\exp \left(-\pi \lambda_{\mathrm{I}} \Gamma\left(1+\frac{2}{\alpha}\right) s^{\frac{2}{\alpha}} \Gamma\left(1-\frac{2}{\alpha}\right)\right) .
\end{aligned}
$$

When $\alpha=4$, the closed form expression for the probability density function (PDF) of $X$ via inverse Laplace transform can be derived as

$$
f_{X}(x)=\frac{\lambda_{\mathrm{I}}}{4} \pi^{\frac{3}{2}} x^{-\frac{3}{2}} \exp \left(-\frac{\lambda_{\mathrm{I}}^{2} \pi^{4}}{16 x}\right), \quad x \geq 0
$$

Since $\left|h_{s, d}\right|^{2} d_{s, d}^{-\alpha}$ in (12) follows an exponential distribution with parameter $d_{s, d}^{\alpha}$, then the outage probability can be expressed as

$$
\tilde{p}_{D T}^{\text {out }}=\mathbb{E}_{X}\left[1-e^{-d_{s, d}^{\alpha}\left(2^{R}-1\right)\left(\frac{P_{n}}{P_{t}}+X\right)}\right] .
$$




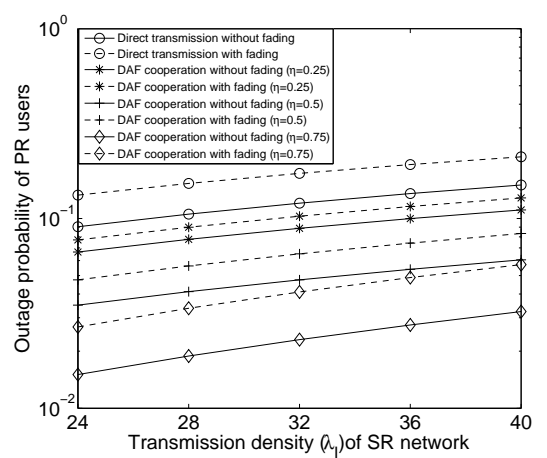

(a) Outage probability of PR users versus transmission density of interferers

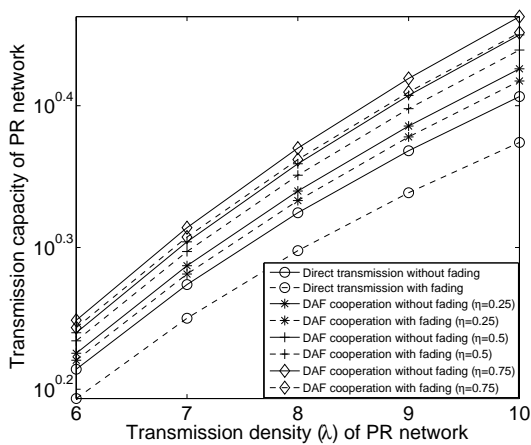

(b) Transmission capacity of PR network versus transmission density

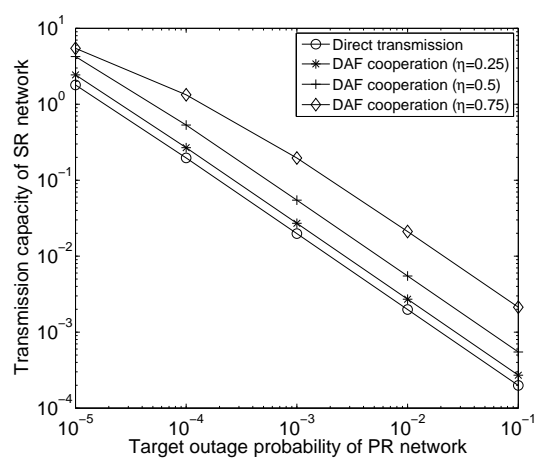

(c) Transmission capacity of SR network versus target outage probability of PR network

Fig. 2. Numerical results

Finally, we have the outage probability for PR users using direct transmission

$$
\tilde{p}_{D T}^{\text {out }}=\frac{\lambda_{\mathrm{I}} \pi^{\frac{3}{2}}}{4}\left(\frac{\Gamma\left(\frac{1}{2}\right)}{\sqrt{\beta}}-e^{-\nu}\left(\frac{\beta}{\gamma}\right)^{-\frac{1}{4}} K_{-\frac{1}{2}}(2 \sqrt{\beta \gamma})\right)
$$

where $\beta=\frac{\lambda_{1}^{2} \pi^{4}}{16}, \nu=d_{s, d}^{\alpha}\left(2^{R}-1\right) \frac{P_{n}}{P_{t}}, \gamma=d_{s, d}^{\alpha}\left(2^{R}-1\right)$, $\Gamma(z)=\int_{0}^{\infty} t^{z-1} e^{-t} d t$ is incomplete gamma function and $K_{\mathrm{v}}(z)$ is modified Bessel function of second kind. The derivation of (16) can be found in Appendix-C. Then the transmission capacity of PR users using direct transmission is given by

$$
\tilde{C}_{D T}=R \lambda\left(1-\tilde{p}_{D T}^{\text {out }}\right) .
$$

\section{B. Cooperative Transmission}

Lemma 3: (J. N. Laneman et al. [9]). Let $w=u+v$, where $u$ and $v$ are independent exponential random variables with parameters $\rho_{u}$ and $\rho_{v}$, respectively. Then the CDF

$$
\begin{aligned}
& F_{W}(w)= \\
& \begin{cases}1-\left[\left(\frac{\rho_{v}}{\rho_{v}-\rho_{u}}\right) e^{-\rho_{u} w}+\left(\frac{\rho_{u}}{\rho_{u}-\rho_{v}}\right) e^{-\rho_{v} w}\right], & \rho_{u} \neq \rho_{v} \\
1-(1+\rho w) e^{-\rho w}, & \rho_{u}=\rho_{v}=\rho\end{cases}
\end{aligned}
$$

Similar as the non-fading case, we derive a closed form expression for cooperative transmission

$$
\begin{aligned}
\tilde{p}_{C T}^{\text {out }}= & \frac{\lambda_{\mathrm{I}}^{2} \pi^{3}}{16}\left(\frac{\Gamma\left(\frac{1}{2}\right)}{\sqrt{\beta}}-e^{-\nu_{\mathrm{A}}}\left(\frac{\beta}{\gamma_{\mathrm{A}}}\right)^{-\frac{1}{4}} K_{-\frac{1}{2}}\left(2 \sqrt{\beta \gamma_{\mathrm{A}}}\right)\right) \\
& \times\left(\frac{\Gamma\left(\frac{1}{2}\right)}{\sqrt{\beta}}-e^{-\nu_{\mathrm{B}}}\left(\frac{\beta}{\gamma_{\mathrm{B}}}\right)^{-\frac{1}{4}} K_{-\frac{1}{2}}\left(2 \sqrt{\beta \gamma_{\mathrm{B}}}\right)\right)+(1- \\
& \left.\frac{\lambda_{\mathrm{I}} \pi^{\frac{3}{2}}}{4}\left(\frac{\Gamma\left(\frac{1}{2}\right)}{\sqrt{\beta}}-e^{-\nu_{\mathrm{A}}}\left(\frac{\beta}{\gamma_{\mathrm{A}}}\right)^{-\frac{1}{4}} K_{-\frac{1}{2}}\left(2 \sqrt{\beta \gamma_{\mathrm{A}}}\right)\right)\right) \times \\
& \frac{\lambda_{\mathrm{I}} \pi^{\frac{3}{2}}}{4}\left(\frac{\Gamma\left(\frac{1}{2}\right)}{\sqrt{\beta}}-\frac{d_{r, d}^{\alpha} e^{-\nu_{\mathrm{D} 1}}\left(\frac{\beta}{\gamma_{\mathrm{D} 1}}\right)^{-\frac{1}{4}}}{d_{r, d}^{\alpha}-d_{s, d}^{\alpha}} K_{-\frac{1}{2}}\left(2 \sqrt{\beta \gamma_{\mathrm{D} 1}}\right)\right. \\
& \left.-\frac{d_{s, d}^{\alpha} e^{-\nu_{\mathrm{D} 2}}\left(\frac{\beta}{\gamma_{\mathrm{D}}}\right)^{-\frac{1}{4}}}{d_{s, d}^{\alpha}-d_{r, d}^{\alpha}} K_{-\frac{1}{2}}\left(2 \sqrt{\beta \gamma_{\mathrm{D} 2}}\right)\right)
\end{aligned}
$$

where $\nu_{\mathrm{A}}=d_{s, r}^{\alpha}\left(2^{2 R}-1\right) \frac{P_{n}}{P_{t}}, \gamma_{\mathrm{A}}=d_{s, r}^{\alpha}\left(2^{2 R}-1\right), \nu_{\mathrm{B}}=$ $\frac{d_{s, d}^{\alpha}}{2}\left(2^{2 R}-1\right) \frac{P_{n}}{P_{t}}, \gamma_{\mathrm{B}}=\frac{d_{s, d}^{\alpha}}{2}\left(2^{2 R}-1\right), \nu_{\mathrm{D} 1}=d_{s, d}^{\alpha}\left(2^{2 R}-1\right) \frac{P_{n}}{P_{t}}$, $\gamma_{\mathrm{D} 1}=d_{s, d}^{\alpha}\left(2^{2 R}-1\right), \nu_{\mathrm{D} 2}=d_{r, d}^{\alpha}\left(2^{2 R}-1\right) \frac{P_{n}}{P_{t}}, \gamma_{\mathrm{D} 2}=d_{r, d}^{\alpha}\left(2^{2 R_{-}}\right.$
1) and $\beta=\frac{\lambda_{1}^{2} \pi^{4}}{16}$. The mathematical detail of (18) is provided in Appendix-D and the transmission capacity of PR users using cooperative transmission is given by

$$
\tilde{C}_{C T}=R \lambda\left(1-\tilde{p}_{C T}^{\text {out }}\right) \text {. }
$$

\section{Numerical RESUlts}

We assume that transmission power for both PR and SR users is $P_{t}=10$, the noise power $P_{n}=1$, the desired data rate $R=0.3 \mathrm{bit} / \mathrm{s} / \mathrm{Hz}$, path loss exponent $\alpha=4$, the transmission distance between a source-destination pair is $d=1$. Motivated by the fact that the best relay is located on the line between the source and destination pair, we choose three different relay candidates in our analysis and use $\eta=\frac{d_{s, r}}{d_{s, d}}$ to define the normalized location of the relay. As can be seen in Fig. 2 (a), the outage probabilities for both cooperative and direct transmission increase monotonically with the transmission density of interferers from the SR network. In addition, PR users employing cooperative transmission achieves much better performance than that employing direct transmission.

By contrast, even though outage performance deteriorates as transmission density rises, transmission capacity shows a upper trend in Fig. 2 (b). Furthermore, it is clear that fading actually has negative effect on system performance in which the outage probability and transmission capacity perform worse than that in non-fading case. Fig. 2 (c) shows the transmission capacity of the SR network in the scenario of different target outage probability achieved by PR users using different schemes. It further verifies Theorem 2 that the use of cooperative transmission in the PR network can also increase transmission capacity of the SR network.

\section{CONCLUSions}

We have employed a stochastic geometry model to analyze the transmission capacity of the Decode-and-Forward (DAF) cooperation scheme in an overlaid wireless network. It has shown that the use of cooperative transmission achieves much better reliability and a larger transmission capacity. Furthermore, such performance gain can be manipulated to increase the capacity of the SR network without deteriorating the performance of the PR network. 


\section{APPENDIX}

\section{A. Derivation of (9)}

Assume that a relay node is randomly selected. Hence the mutual information of this cooperative link can be shown as

$$
\mathbf{I}_{C}= \begin{cases}\frac{1}{2} \log \left(1+2 \operatorname{SINR}_{s, d}\right), & \operatorname{SINR}_{s, r}<q(R) \\ \frac{1}{2} \log \left(1+\operatorname{SINR}_{s, d}+\operatorname{SINR}_{r, d}\right), & \operatorname{SINR}_{s, r} \geq q(R)\end{cases}
$$

Therefore, the outage probability for the Selected Decodeand-Forward (SDF) is given by $\mathrm{I}_{C}<R$ and is a sum

$$
\begin{aligned}
& p_{C T}^{\text {out }}=\operatorname{Pr}\left[\mathrm{I}_{C}<R\right] \\
& =\underbrace{\operatorname{Pr}\left[\operatorname{SINR}_{s, r}<q(R)\right]}_{A} \underbrace{\operatorname{Pr}\left[2 \operatorname{SINR}_{s, d}<q(R)\right]}_{B} \\
& +\underbrace{\operatorname{Pr}\left[\operatorname{SINR}_{s, r} \geq q(R)\right]}_{C} \underbrace{\operatorname{Pr}\left[\operatorname{SINR}_{s, d}+\operatorname{SINR}_{r, d}<q(R)\right]}_{D}
\end{aligned}
$$

By computing each component above, we have

$$
A=1-2 Q\left(\frac{\pi^{\frac{3}{2}} \lambda_{\mathrm{I}}}{\sqrt{2 \varepsilon_{1}}}\right), B=1-2 Q\left(\frac{\pi^{\frac{3}{2}} \lambda_{\mathrm{I}}}{\sqrt{2 \varepsilon_{2}}}\right), C=1-A
$$

where $\varepsilon_{1}=\frac{d_{s, r}^{-\alpha}}{2^{2 R}-1}-\frac{P_{n}}{P_{t}}$ and $\varepsilon_{2}=\frac{2 d_{s, d}^{-\alpha}}{2^{2 R}-1}-\frac{P_{n}}{P_{t}}$. The calculations in (20) are analogous to III-A and can be directly derived from direct transmission.

According to the time schedule assumed in Section II, the interferers which are mainly from SR users keep the same with regard to a PR receiver during one time slot transmission $(\mathrm{T})$, $D$ therefore can be derived as

$$
D=\operatorname{Pr}\left[\frac{P_{t}\left(d_{s, d}^{-\alpha}+d_{r, d}^{-\alpha}\right)}{P_{n}+\sum_{i \in \Phi} P_{t}\left|X_{i}\right|^{-\alpha}} \leq q(R)\right]=1-2 Q\left(\frac{\pi^{\frac{3}{2}} \lambda_{\mathrm{I}}}{\sqrt{2 \varepsilon_{3}}}\right)
$$

where $\varepsilon_{3}=\frac{d_{s, d}^{-\alpha}+d_{r, d}^{-\alpha}}{2^{2 R}-1}-\frac{P_{n}}{P_{t}}$.

\section{B. Proof for Theorem 1}

1) Direct transmission: For Q function, it has the relationship $Q(x)=\frac{1}{2}-\frac{1}{2} \operatorname{erf}\left(\frac{x}{\sqrt{2}}\right)$. Then, the outage probability for direct transmission in (6) can be converted to $p_{D T}^{\text {out }}=\operatorname{erf}\left(\frac{\pi^{\frac{3}{2}} \lambda_{I}}{2 \sqrt{\varepsilon}}\right)$. Assume the outage probability $p_{D T}^{\text {out }} \rightarrow 0$, by using the Taylor

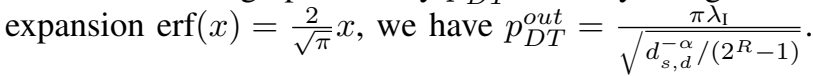

2) Cooperative transmission: Similar to the direct transmission above, we can derive the outage probability for cooperative transmission $p_{C T}^{\text {out }}=\frac{\pi \lambda_{\mathrm{I}}}{\sqrt{\frac{d_{s, d}^{-\alpha}+d_{r, d}^{-\alpha}}{2^{2 R}-1}}}+o\left(\left(\frac{\pi \lambda_{\mathrm{I}}}{\sqrt{\frac{d-\alpha}{2^{2 R}-1}}}\right)^{2}\right)$, since the outage probability is very small, the second component is reduced with a higher order than the first component. The ratio $g$ is derived as $g=\frac{p_{C T}^{\text {out }}}{p_{D T}^{\text {out }}} \approx \sqrt{\frac{d_{s, d}^{-\alpha}\left(2^{R}+1\right)}{d_{s, d}^{-\alpha}+d_{r, d}^{-\alpha}}}$.

\section{Derivation of (16)}

The outage probability of the direct transmission in (15) can be derived as $\tilde{p}_{D T}^{\text {out }}=\mathbb{E}_{X}\left[1-e^{-d_{s, d}^{\alpha}\left(2^{R}-1\right)\left(\frac{P_{n}}{P_{t}}+X\right)}\right]$, where the probability density function of $X$ is $f_{X}(x)=$ $\frac{\lambda_{1}}{4} \pi^{\frac{3}{2}} x^{-\frac{3}{2}} \exp \left(-\frac{\lambda_{1}^{2} \pi^{4}}{16 x}\right)$. Then we have

$$
\begin{aligned}
\tilde{p}_{D T}^{o u t} & =\int_{0}^{\infty} \frac{\lambda_{\mathrm{I}}}{4} \pi^{\frac{3}{2}} x^{-\frac{3}{2}} e^{-\frac{\lambda_{1}^{2} \pi^{4}}{16 x}}\left[1-e^{-d_{s, d}^{\alpha}\left(2^{R}-1\right)\left(\frac{P_{n}}{P_{t}}+x\right)}\right] d x \\
& =\frac{\lambda_{\mathrm{I}}}{4} \pi^{\frac{3}{2}} \int_{0}^{\infty} \underbrace{x^{-\frac{3}{2}} e^{-\frac{\beta}{x}}}_{\mathrm{I}_{1}}-\underbrace{x^{-\frac{3}{2}} e^{-\frac{\beta}{x}-\nu-\gamma x}}_{\mathrm{I}_{2}} d x
\end{aligned}
$$

where $\beta=\frac{\lambda_{1}^{2} \pi^{4}}{16}, \nu=d_{s, d}^{\alpha}\left(2^{R}-1\right) \frac{P_{n}}{P_{t}}$ and $\gamma=d_{s, d}^{\alpha}\left(2^{R}-1\right)$ and have

$$
\mathrm{I}_{1}=\frac{\lambda_{\mathrm{I}}}{4} \pi^{\frac{3}{2}} \int_{0}^{\infty} x^{-\frac{3}{2}} e^{-\frac{\beta}{x}} d x=\frac{\lambda_{\mathrm{I}}}{4} \pi^{\frac{3}{2}} \frac{1}{\sqrt{\beta}} \Gamma\left(\frac{1}{2}\right),
$$

According to [10], we can derive the following for part $\mathrm{I}_{2}$

$$
\mathrm{I}_{2}=\frac{\lambda_{\mathrm{I}}}{2} \pi^{\frac{3}{2}} e^{-\nu}\left(\frac{\beta}{\gamma}\right)^{-\frac{1}{4}} K_{-\frac{1}{2}}(2 \sqrt{\beta \gamma}) .
$$

\section{Derivation of (18)}

The components $A, B, C$ in (20) can be derived similarly as direct transmission. According to Lemma 3, since $\xi_{1}=\left|h_{s, d}\right|^{2} d_{s, d}^{-\alpha}$ and $\xi_{2}=\left|h_{r, d}\right|^{2} d_{r, d}^{-\alpha}$ are two independent exponential distributed random variables with parameter $d_{s, d}^{\alpha}$ and $d_{r, d}^{\alpha}$, respectively, the form of $D$ is analogous to (15) and can be directly derived from Appendix- $\mathrm{C}$ as

$$
\begin{aligned}
D= & \frac{\lambda_{\mathrm{I}} \pi^{\frac{3}{2}}}{4}\left(\frac{\Gamma\left(\frac{1}{2}\right)}{\sqrt{\beta}}-\frac{d_{r, d}^{\alpha} e^{-\nu_{\mathrm{D} 1}}\left(\frac{\beta}{\gamma_{\mathrm{D} 1}}\right)^{-\frac{1}{4}}}{d_{r, d}^{\alpha}-d_{s, d}^{\alpha}} K_{-\frac{1}{2}}\left(2 \sqrt{\beta \gamma_{\mathrm{D} 1}}\right)\right. \\
& \left.-\frac{d_{s, d}^{\alpha} e^{-\nu_{\mathrm{D} 2}}\left(\frac{\beta}{\gamma_{\mathrm{D} 2}}\right)^{-\frac{1}{4}}}{d_{s, d}^{\alpha}-d_{r, d}^{\alpha}} K_{-\frac{1}{2}}\left(2 \sqrt{\beta \gamma_{\mathrm{D} 2}}\right)\right) .
\end{aligned}
$$

\section{REFERENCES}

[1] P. Gupta and P. Kumar, "The capacity of wireless networks," IEEE Transactions on Information Theory, vol. 46, pp. 388-404, March 2000.

[2] S. P. Weber, X. Yang, J. G. Andrews, and G. de Veciana, "Transmission capacity of wireless ad hoc networks with outage constraints," IEEE Transactions on Information Theory, vol. 51, pp. 4091-4102, December 2005.

[3] S. Weber, J. Andrews, and N. Jindal, "The effect of fading, channel inversion, and threshold scheduling on ad hoc networks," Information Theory, IEEE Transactions on, vol. 53, no. 11, pp. 4127-4149, Nov. 2007.

[4] S. Toumpis and A. J. Goldsmith, "Capacity regions for wireless ad hoc networks," IEEE Transactions on Wireless Communication, vol. 24, pp. 736-748, May 2003.

[5] C. Yin, L. Gao, T. Liu, and S. Cui, "Transmission capacities for overlaid wireless ad hoc networks with outage constraints," in IEEE International Conference on Communications (ICC), Germany, 2009.

[6] N. Abramson, "The aloha system- another alternative for computer communication," in Proc. AFIPS, 1970.

[7] J. Venkataraman, M. Haenggi, and O. Collins, "Shot noise models for outage and throughput analyses in wireless ad hoc networks," in IEEE Military Communications Conference, October 2006, pp. 1-7.

[8] J. N. Laneman, D. N. Tse, and G. W. Wornell, "Cooperative diversity in wireless networks: Efficient protocols and outage behavior," IEEE Trans. On Information Theory, vol. 50(12), Dec. 2004.

[9] J. N. Laneman and G. W. Wornell, "Distributed space-time-coded protocols for exploiting cooperative diversity in wireless networks," IEEE Trans. Information Theory, vol. 49, pp. 2415-2425, Oct. 2003.

[10] I. S. Gradshtein and R. S., Table Of Integrals, Series And Products, sixth edition. Elsevier Inc., 2000. 